\title{
Reconstructing the vertical distribution of the aeolian saltation mass flux based on the probability distribution of lift-off velocity
}

\author{
Liqiang Kang ${ }^{\mathrm{a}, \mathrm{b}, *}$, Liejin Guo ${ }^{\mathrm{b}}$, Dayou Liu ${ }^{\mathrm{a}}$ \\ ${ }^{a}$ Division of Engineering Sciences, Institute of Mechanics, Chinese Academy of Sciences, Beijing 100080, China \\ ${ }^{\mathrm{b}}$ State Key Laboratory of Multiphase Flow in Power Engineering, Xi'an Jiaotong University, Xi'an 710049, China
}

Received 30 October 2006; received in revised form 4 July 2007; accepted 6 July 2007

Available online 24 July 2007

\begin{abstract}
The probability distribution of lift-off velocity of the saltating grains is a bridge to linking microscopic and macroscopic research of aeolian sand transport. The lift-off parameters of saltating grains (i.e., the horizontal and vertical lift-off velocities, resultant lift-off velocity, and lift-off angle) in a wind tunnel are measured by using a Phase Doppler Particle Analyzer (PDPA). The experimental results show that the probability distribution of horizontal lift-off velocity of saltating particles on a bed surface is a normal function, and that of vertical lift-off velocity is an exponential function. The probability distribution of resultant lift-off velocity of saltating grains can be expressed as a log-normal function, and that of lift-off angle complies with an exponential function. A numerical model for the vertical distribution of aeolian mass flux based on the probability distribution of lift-off velocity is established. The simulation gives a sand mass flux distribution which is consistent with the field data of Namikas (Namikas, S.L., 2003. Field measurement and numerical modelling of aeolian mass flux distributions on a sandy beach, Sedimentology 50, 303-326). Therefore, these findings are helpful to further understand the probability characteristics of lift-off grains in aeolian sand transport.
\end{abstract}

(C) 2007 Elsevier B.V. All rights reserved.

Keywords: Aeolian saltation; Lift-off velocity; Probability distribution; Vertical distribution of mass flux

\section{Introduction}

Aeolian sand transport is an important geomorphological process for landform change in natural environments including deserts and sandy beaches on the Earth, and it even occurs on other planets such as Mars, possibly Venus and Titan (Greeley and Iversen, 1985). It also causes some environmental problems, for example,

\footnotetext{
* Corresponding author. Division of Engineering Sciences, Institute of Mechanics, Chinese Academy of Sciences, Beijing 100080, China. Tel.: +8610 82544226; fax: +861062561284.

E-mail address: klq@sohu.com (L. Kang).
}

soil erosion, sand storms and desertification. Sand transport by wind is a special case of gas-solid twophase flow. The processes occurring in blown sand transport are creep, saltation and suspension, of which saltation, where sand grains are propelled by wind along the surface in short hops, is the dominant mode of blown sand movement, accounting for about $75 \%$ of the total sand flux (Bagnold, 1941). The lift-off velocity probability distribution of the saltating grains is a bridge to linking the microscopic and macroscopic aeolian research. It is important to accurately describe the lift-off velocity distribution of saltating grains, which is helpful in estimating the statistical parameters of saltating grains 
from each representative particle trajectory. Since the classic work of Bagnold (1941), much empirical and theoretical research has greatly improved our understanding of aeolian sand transport, but the probability characteristics of entrained grains on a bed surface in the saltation process are not well understood and further studies are needed.

By using wind tunnel experimentation and numerical modeling, a great deal of effort has been contributed to studying the entrainment parameters such as the horizontal and vertical lift-off velocities, resultant liftoff velocity and angle (e.g. White and Schulz, 1977; Willetts and Rice, 1986; Anderson and Hallet, 1986; Werner and Haff, 1988; Anderson and Haff, 1988; Sørensen, 1991; Haff and Anderson, 1993; Nalpanis et al., 1993; Rice et al., 1995, 1996; Dong et al., 2002a; Namikas, 2003). If the probability distributions of the horizontal and vertical lift-off velocities or the resultant lift-off velocity and angle of saltating grains are known, the proportion of the number of grains with a given resultant lift-off velocity and lift-off angle will be acquired, then the macroscopic statistic of particles can be deduced from the representative trajectories of saltating grains. Sørensen (1991) used a normal function to describe the distribution of the sand horizontal ejection velocity. Raupach (1991) and Anderson and Hallet (1986) described the initial vertical velocity by an exponential distribution. Anderson and Haff (1988, 1991) used a Gaussian distribution for the rebound velocity of particles, and used an exponential function for the ejected velocity. Nalpanis et al. (1993) found that the probability distribution of resultant lift-off velocity and angle is similar to the log-normal distribution. Dong et al. (2002a) gave experimental results showing that the probability distribution of the velocity of entrained particles is best described by a Weibull function. Namikas (2003) used the gamma and exponential launch velocity distributions in the numerical model.

The grain-bed collision process determines the launch velocity and angle of blown sands on a bed surface, which further affects particle trajectories, wind field and sand flux. Most observations of sand movement on a bed surface are from high-speed photography in a wind tunnel (e.g., Mitha et al., 1986; Willetts and Rice, 1986; Rice et al., 1995, 1996; Nalpanis et al., 1993). In general, the grain-bed collision process is difficult to measure directly from the high-speed photography because the grains are crowded at the very low height (e.g., Nalpanis et al., 1993; Zou et al., 2001). But recently, by using the advanced high-speed CMOS camera with very high frame rate of $2000 \mathrm{fps}$, Zhang et al. $(2007 \mathrm{a}, \mathrm{b})$ captured the trajectories and velocities of sand grains in the region very close to the ground surface at the lower free-stream wind velocity (less than $10 \mathrm{~m} / \mathrm{s}$ ).

A natural sand bed is generally composed of variably sized and shaped grains, and has many different geometric configurations. The incident grains colliding with the bed also include a variety of grain shape and size. The complexity of the problem is apparent. Therefore, the theoretical models of the grain-bed collision process are usually restricted to the simple conditions. In some numerical models, the grain-bed collision is an important component and generally treated in an empirical way, the splash function (Anderson and Haff, 1988,1991; Ungar and Haff, 1987) or a set of the experimental data (McEwan and Willetts, 1991, 1993) is used to describe the collision outcomes. Haff and Anderson (1993) studied the grain-bed collision by a particle dynamics method, and gave the statistical representations of the splash process. Ta and Dong (2007) applied the theory of cascade collision to simulate the sand grain-bed collision process. These theoretical models improved our understanding of the complex grain-bed collision process in windblown sand movement. However, the detailed movement mechanism of entrained grains still remains uncertain.

In the present work, the probability characteristics of lift-off grains on a bed surface are measured by using Phase Doppler Particle Analyzer (PDPA), which is a non-intrusive measurement using an optical technique and can acquire the objective statistical results of many particles. Then a numerical model combined with the lift-off velocity probability distribution is established to reconstruct the vertical distribution of the aeolian saltation mass flux. The simulated mass flux is compared with the field data of Namikas (2003). In Sections 2 and 3 , the experimental methods and results for the lift-off velocity probability distribution are reported. The numerical methods and analysis are shown in Sections 4,5 and 6 . The primary object of this work is to reveal the lift-off velocity probability distribution of the saltating grains in the entrainment process, i.e., the probability distributions of horizontal and vertical lift-off velocities, resultant lift-off velocity and lift-off angle.

\section{Experimental methods for lift-off velocity distribution}

The experiment is performed in a sand wind tunnel at the State Key Laboratory of Multiphase Flow in Power Engineering, Xi' an Jiaotong University. The wind tunnel is a blow-type non-circulating wind tunnel. The working section of wind tunnel is $12 \mathrm{~m}$ long with a cross-section that is $0.4 \mathrm{~m}$ wide and $0.6 \mathrm{~m}$ high. The 
free-stream wind velocity in the wind tunnel can be changed continuously from 1 to $40 \mathrm{~m} / \mathrm{s}$.

The natural quartz sand was sieved into three size groups: $0.17-0.30,0.30-0.36$, and $0.36-0.44 \mathrm{~mm}$. The layer of sand samples is about $4 \mathrm{~m}$ long, $0.4 \mathrm{~m}$ wide and $3-4 \mathrm{~cm}$ deep, and the sand bed surface is leveled to the wind tunnel floor. The free-stream wind velocity is measured by a hot wire anemometer at the start of the sand bed layer.

The movement of entrained grains in the saltation process is measured by using a Phase Doppler Particle Analyzer (PDPA), a TSI apparatus, at about $1 \mathrm{~mm}$ above the sand bed surface near the downwind edge of the sand layer. The PDPA has many advantages. It is a nonintrusive measurement using an optical technique. It has a very high accuracy, the measurement accuracy for particle velocity is within $0.3 \%$. It can also give the objective statistical results of many particles. The PDPA parameters in the experiments are listed in Table 1.

The layout of wind tunnel and PDPA is shown in Fig. 1. The glass window of wind tunnel provides optical access for the PDPA to produce the laser beams into the wind tunnel. A computer records the horizontal and vertical velocities of the moving particles for further analysis.

The experimental procedure for each test is performed as follows:

(1) The layer of sand samples is put on the tunnel floor.

(2) Turn on the hot wire anemometer and the PDPA.

(3) Adjust the 3-axis traverse system, and make the PDPA probe volume to the measurement position.

(4) Turn on the wind tunnel.

(5) The hot wire anemometer is used to measure the free-stream wind velocity.

Table 1

The PDPA parameters in the experiments

\begin{tabular}{ll}
\hline Item & Specification \\
\hline Transmitting lens focal length & $600 \mathrm{~mm}$ \\
Wavelength & $514.5 \mathrm{~nm}$ (green) \\
& $488 \mathrm{~nm}$ (blue) \\
Beam separation & $25 \mathrm{~mm}$ (green) \\
& $50 \mathrm{~mm}$ (blue) \\
Laser beam diameter & $1.8 \mathrm{~mm}$ (green) \\
& $1.8 \mathrm{~mm}$ (blue) \\
Fringe spacing & $12.35 \mu \mathrm{m}$ (green) \\
Probe volume length & $5.86 \mu \mathrm{m}$ (blue) \\
& $10.484 \mathrm{~mm}$ (green) \\
Waist diameter & $4.975 \mathrm{~mm}$ (blue) \\
& $0.218 \mathrm{~mm}$ (green) \\
\hline
\end{tabular}

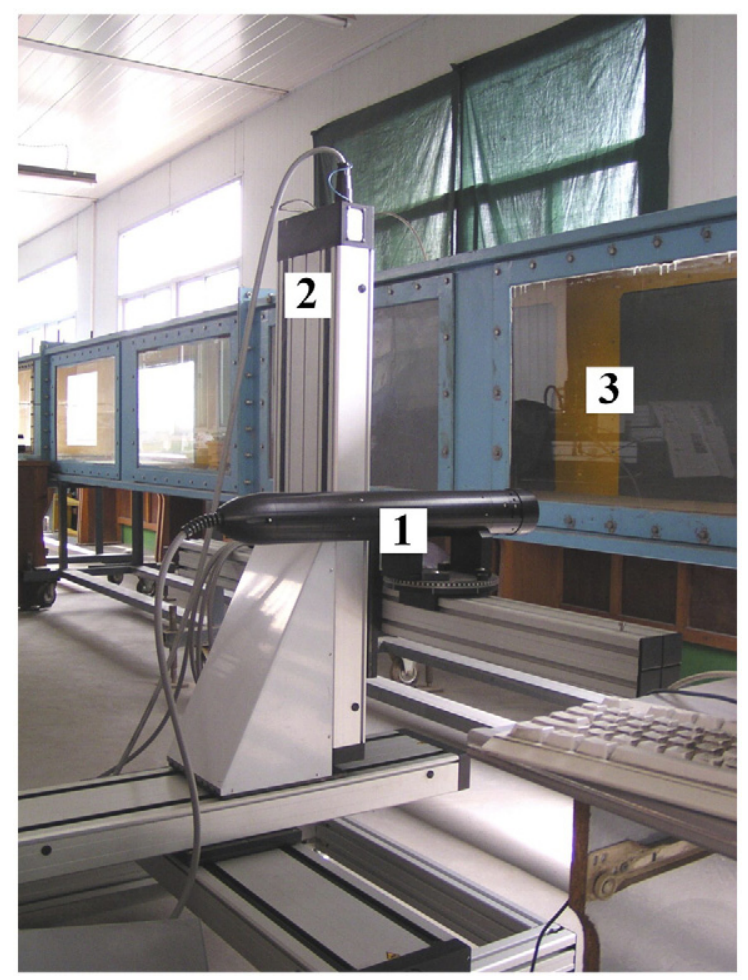

Fig. 1. The layout of wind tunnel and PDPA. (1. Transmitting probe of PDPA; 2. 3-axis traverse system; 3. Glass window of the wind tunnel).

(6) The PDPA is used to measure the particle velocity.

(7) Turn off the wind tunnel, hot wire anemometer and PDPA.

The above procedure was repeated until all tests were finished. There are total 13 sets of wind velocity and corresponding lift-off sand grain data. The data acquisition of the PDPA was set in advance to measure 3000 validated particles or the number of validated particles in 30s for series No. 1, 2 and 4 in Tables 2-5, 20s for series No. 3 and 9, and 15 s for all other series.

\section{Experimental results and discussion for lift-off velocity distribution}

\subsection{The horizontal and vertical lift-off velocity distributions of the saltating grains}

We can distinguish the lift-off particles from other particles in a saltating cloud by the vertical velocity. If the vertical velocity of a particle is upward, the particle will be ascending and is referred to as the lift-off particle.

The probability density distribution of horizontal liftoff velocity of the entrained particles on bed surface is shown on Fig. 2. In Fig. $2, d_{p}$ is sand diameter, and $u_{f 0}$ is 
Table 2

Fitting parameters and Kolmogorov-Smirnov test of horizontal lift-off velocity distribution of the saltating grains

\begin{tabular}{|c|c|c|c|c|c|c|c|c|c|c|c|}
\hline \multirow{2}{*}{$\begin{array}{l}\text { Serial } \\
\text { no. }\end{array}$} & \multirow[t]{2}{*}{$d_{p}(\mathrm{~mm})$} & \multirow[t]{2}{*}{$u_{f 0}(\mathrm{~m} / \mathrm{s})$} & \multicolumn{5}{|c|}{ Fitting parameters } & \multicolumn{4}{|c|}{ Kolmogorov-Smirnov test } \\
\hline & & & $A$ & Error of $A$ & $B$ & Error of $B$ & $R^{2}$ & $\begin{array}{l}\text { Number of } \\
\text { observations }\end{array}$ & $D_{n}$ & $D_{n, 0.01}$ & \\
\hline 1 & $0.17-0.30$ & 10.9 & 0.5318 & 0.0183 & 0.5844 & 0.0223 & 0.947 & 219 & 0.088 & 0.11 & Accept $H_{0}$ \\
\hline 2 & & 11.6 & 0.503 & 0.0192 & 0.7135 & 0.0235 & 0.942 & 257 & 0.077 & 0.1 & Accept $H_{0}$ \\
\hline 3 & & 13.5 & 0.4821 & 0.0134 & 0.5951 & 0.0164 & 0.967 & 286 & 0.075 & 0.096 & Accept $H_{0}$ \\
\hline 4 & & 14.2 & 0.4635 & 0.0088 & 0.5288 & 0.0108 & 0.985 & 793 & 0.041 & 0.058 & Accept $H_{0}$ \\
\hline 5 & & 15.5 & 0.4471 & 0.0159 & 0.5142 & 0.0194 & 0.945 & 126 & 0.096 & 0.15 & Accept $H_{0}$ \\
\hline 6 & $0.30-0.36$ & 11.1 & 0.4228 & 0.0114 & 0.4386 & 0.0139 & 0.97 & 323 & 0.079 & 0.091 & Accept $H_{0}$ \\
\hline 7 & & 12.3 & 0.4713 & 0.0125 & 0.4784 & 0.0153 & 0.97 & 302 & 0.064 & 0.094 & Accept $H_{0}$ \\
\hline 8 & & 14.8 & 0.4207 & 0.0132 & 0.5515 & 0.0161 & 0.959 & 224 & 0.076 & 0.11 & Accept $H_{0}$ \\
\hline 9 & $0.36-0.44$ & 12.3 & 0.3801 & 0.0139 & 0.3831 & 0.017 & 0.94 & 500 & 0.11 & 0.073 & Reject $H_{0}$ \\
\hline 10 & & 13.2 & 0.4128 & 0.0117 & 0.4975 & 0.0142 & 0.967 & 318 & 0.083 & 0.091 & Accept $H_{0}$ \\
\hline 11 & & 14.4 & 0.4381 & 0.0137 & 0.4229 & 0.0167 & 0.96 & 374 & 0.08 & 0.084 & Accept $H_{0}$ \\
\hline 12 & & 15.8 & 0.4329 & 0.0137 & 0.6134 & 0.0167 & 0.959 & 269 & 0.061 & 0.099 & Accept $H_{0}$ \\
\hline 13 & & 17.2 & 0.6216 & 0.0137 & 0.6677 & 0.0168 & 0.978 & 373 & 0.038 & 0.084 & Accept $H_{0}$ \\
\hline
\end{tabular}

$d_{p}$ is sand diameter; $u_{f 0}$ is the free-stream wind velocity; $\mathrm{A}$ and $\mathrm{B}$ are the regression coefficients; $R^{2}$ is the correlation coefficient.

$D_{n}$ is test statistic; $D_{n, 0.01}$ is critical value of test statistic; $H_{0}$ : The sample data follow a normal distribution.

the free-stream wind velocity. It can be seen that the horizontal lift-off velocity distribution of the sand particles has a typical peak at the different free-stream wind velocities.

By using the least squares curve-fit method, the probability density function of horizontal lift-off velocity of the entrained particles complies with a normal distribution, as follows:

$P\left(u_{p l}\right)=\frac{1}{\sqrt{2 \pi} A} \exp \left(-\frac{\left(u_{p l}-B\right)^{2}}{2 A^{2}}\right)$

where $P\left(u_{p l}\right)$ is the probability density, $u_{p l}$ is the horizontal lift-off velocity of the entrained grains in meters per second. $A$ and $B$ are the regression coefficients.

Fig. 3 denotes the probability density function of vertical lift-off velocity of the saltating grains, which can be described by an exponential function:

$P\left(v_{p l}\right)=\frac{1}{A} \exp \left(-\frac{v_{p l}}{A}\right)$

where $P\left(v_{p l}\right)$ is the probability density, $v_{p l}$ is the vertical lift-off velocity of the saltating grains in meters per second. $A$ is the regression coefficient.

Table 2 lists the fitting parameters for horizontal liftoff velocity distribution of the saltating grains. From Table 2 the correlation is reasonably good $\left(R^{2}>=0.94\right)$.

Table 3

Fitting parameters and Kolmogorov-Smirnov test of vertical lift-off velocity distribution of the saltating grains

\begin{tabular}{|c|c|c|c|c|c|c|c|c|c|}
\hline \multirow{2}{*}{$\begin{array}{l}\text { Serial } \\
\text { no. }\end{array}$} & \multirow[t]{2}{*}{$d_{p}(\mathrm{~mm})$} & \multirow{2}{*}{$\begin{array}{l}u_{f 0} \\
(\mathrm{~m} / \mathrm{s})\end{array}$} & \multicolumn{3}{|c|}{ Fitting parameters } & \multicolumn{4}{|l|}{ Kolmogorov-Smirnov test } \\
\hline & & & $A$ & Error of $A$ & $R^{2}$ & Number of observations & $D_{n}$ & $D_{n, 0.01}$ & \\
\hline 1 & $0.17-0.30$ & 10.9 & 0.4348 & 0.025 & 0.936 & 219 & 0.058 & 0.11 & Accept $H_{0}$ \\
\hline 2 & & 11.6 & 0.3952 & 0.0182 & 0.96 & 257 & 0.045 & 0.1 & Accept $H_{0}$ \\
\hline 3 & & 13.5 & 0.3977 & 0.017 & 0.967 & 286 & 0.078 & 0.096 & Accept $H_{0}$ \\
\hline 4 & & 14.2 & 0.3784 & 0.0148 & 0.974 & 795 & 0.0581 & 0.0578 & Reject $H_{0}$ \\
\hline 5 & & 15.5 & 0.3903 & 0.0329 & 0.899 & 126 & 0.084 & 0.15 & Accept $H_{0}$ \\
\hline 6 & $0.30-0.36$ & 11.1 & 0.3544 & 0.0175 & 0.968 & 323 & 0.037 & 0.091 & Accept $H_{0}$ \\
\hline 7 & & 12.3 & 0.3461 & 0.0128 & 0.983 & 302 & 0.05 & 0.094 & Accept $H_{0}$ \\
\hline 8 & & 14.8 & 0.412 & 0.021 & 0.958 & 224 & 0.043 & 0.11 & Accept $H_{0}$ \\
\hline 9 & $0.36-0.44$ & 12.3 & 0.3244 & 0.0121 & 0.983 & 500 & 0.041 & 0.073 & Accept $H_{0}$ \\
\hline 10 & & 13.2 & 0.3663 & 0.0179 & 0.968 & 318 & 0.057 & 0.091 & Accept $H_{0}$ \\
\hline 11 & & 14.4 & 0.3535 & 0.0175 & 0.96 & 374 & 0.054 & 0.084 & Accept $H_{0}$ \\
\hline 12 & & 15.8 & 0.3956 & 0.0125 & 0.982 & 269 & 0.07 & 0.099 & Accept $H_{0}$ \\
\hline 13 & & 17.2 & 0.3727 & 0.0187 & 0.96 & 373 & 0.059 & 0.084 & Accept $H_{0}$ \\
\hline
\end{tabular}

$A$ is the regression coefficient; $R^{2}$ is the correlation coefficient.

$D_{n}$ is test statistic; $D_{n, 0.01}$ is critical value of test statistic; $H_{0}$ : The sample data follow an exponential distribution. 
Table 4

Fitting parameters and Kolmogorov-Smirnov test of the distribution of resultant lift-off velocity of the saltating grains

\begin{tabular}{|c|c|c|c|c|c|c|c|c|c|c|c|}
\hline \multirow{2}{*}{$\begin{array}{l}\text { Serial } \\
\text { no. }\end{array}$} & \multirow[t]{2}{*}{$d_{p}(\mathrm{~mm})$} & \multirow{2}{*}{$\begin{array}{l}u_{f 0} \\
(\mathrm{~m} / \mathrm{s})\end{array}$} & \multicolumn{5}{|c|}{ Fitting parameters } & \multicolumn{4}{|l|}{ Kolmogorov-Smirnov test } \\
\hline & & & $A$ & Error of $A$ & $B$ & Error of $B$ & $R^{2}$ & Number of observations & $D_{n}$ & $D_{n, 0.01}$ & \\
\hline 1 & $0.17-0.30$ & 10.9 & 0.6553 & 0.0275 & 0.8613 & 0.0284 & 0.911 & 218 & 0.052 & 0.11 & Accept $H_{0}$ \\
\hline 2 & & 11.6 & 0.6009 & 0.0311 & 0.8873 & 0.033 & 0.881 & 256 & 0.063 & 0.1 & Accept $H_{0}$ \\
\hline 3 & & 13.5 & 0.6132 & 0.0227 & 0.7898 & 0.0215 & 0.938 & 286 & 0.053 & 0.096 & Accept $H_{0}$ \\
\hline 4 & & 14.2 & 0.6552 & 0.0174 & 0.7273 & 0.0152 & 0.968 & 791 & 0.062 & 0.058 & Reject $H_{0}$ \\
\hline 5 & & 15.5 & 0.6908 & 0.044 & 0.7718 & 0.0407 & 0.83 & 126 & 0.096 & 0.15 & Accept $H_{0}$ \\
\hline 6 & $0.30-0.36$ & 11.1 & 0.6931 & 0.0299 & 0.6806 & 0.0244 & 0.92 & 323 & 0.064 & 0.091 & Accept $H_{0}$ \\
\hline 7 & & 12.3 & 0.7449 & 0.0314 & 0.696 & 0.0259 & 0.925 & 302 & 0.08 & 0.094 & Accept $H_{0}$ \\
\hline 8 & & 14.8 & 0.5819 & 0.0306 & 0.7917 & 0.029 & 0.882 & 224 & 0.055 & 0.11 & Accept $H_{0}$ \\
\hline 9 & $0.36-0.44$ & 12.3 & 0.6738 & 0.018 & 0.5917 & 0.0127 & 0.968 & 500 & 0.047 & 0.073 & Accept $H_{0}$ \\
\hline 10 & & 13.2 & 0.5855 & 0.0147 & 0.7001 & 0.0124 & 0.971 & 318 & 0.042 & 0.091 & Accept $H_{0}$ \\
\hline 11 & & 14.4 & 0.6511 & 0.0222 & 0.6546 & 0.0174 & 0.95 & 374 & 0.048 & 0.084 & Accept $H_{0}$ \\
\hline 12 & & 15.8 & 0.5883 & 0.0282 & 0.8015 & 0.0272 & 0.904 & 269 & 0.07 & 0.099 & Accept $H_{0}$ \\
\hline 13 & & 17.2 & 0.706 & 0.0306 & 0.88 & 0.0322 & 0.905 & 372 & 0.085 & 0.0845 & Reject $H_{0}$ \\
\hline
\end{tabular}

$A$ and $B$ are the regression coefficients; $R^{2}$ is the correlation coefficient.

$D_{n}$ is test statistic; $D_{n, 0.01}$ is critical value of test statistic; $H_{0}$ : The sample data follow a log-normal distribution.

Table 3 gives the curve-fit parameters of vertical lift-off velocity distribution, and shows the correlation coefficient of most of the fitting curves exceeds 0.95 .

In the following, the distribution pattern of the experimental data is also analyzed by the KolmogorovSmirnov goodness-of-fit test. This test can be used to determine whether a sample comes from a specific distribution and is defined as follows:

Null hypothesis $H_{0}$ : the sample data follow a specified distribution.

Alternative hypothesis $\mathrm{H}_{1}$ : the sample data do not follow the specified distribution.
The Kolmogorov-Smirnov test statistic is:

$D_{n}=\sup _{1 \leq i \leq n}\left|F^{*}\left(x_{i}\right)-F_{0}\left(x_{i}\right)\right|$

where $D_{n}$ is the test statistic, $F^{*}(x)$ is the empirical cumulative distribution from the experimental sample data, $F_{0}(x)$ is the theoretical cumulative distribution corresponding with the present curve-fit probability density function, $x_{i}$ is the sample data, and $n$ is the size of sample.

$D_{n, \alpha}$ is the critical value of the test statistic at the level of significance $\alpha$. If $D_{n}>D_{n, \alpha}$, the null hypothesis $H_{0}$ will be rejected. In the present work, the significance

Table 5

Fitting parameters and Kolmogorov-Smirnov test of the distribution of lift-off angle of the saltating grains

\begin{tabular}{|c|c|c|c|c|c|c|c|c|c|}
\hline \multirow{2}{*}{$\begin{array}{l}\text { Serial } \\
\text { no. }\end{array}$} & \multirow[t]{2}{*}{$d_{p}(\mathrm{~mm})$} & \multirow[t]{2}{*}{$u_{f 0}(\mathrm{~m} / \mathrm{s})$} & \multicolumn{3}{|c|}{ Fitting parameters } & \multicolumn{4}{|l|}{ Kolmogorov-Smirnov test } \\
\hline & & & $A$ & Error of $A$ & $R^{2}$ & Number of observations & $D_{n}$ & $D_{n, 0.01}$ & \\
\hline 1 & $0.17-0.30$ & 10.9 & 39.1 & 3.1 & 0.839 & 218 & 0.045 & 0.11 & Accept $H_{0}$ \\
\hline 2 & & 11.6 & 30.9 & 1.5 & 0.939 & 256 & 0.042 & 0.1 & Accept $H_{0}$ \\
\hline 3 & & 13.5 & 36.6 & 2.4 & 0.885 & 286 & 0.041 & 0.096 & Accept $H_{0}$ \\
\hline 4 & & 14.2 & 40.8 & 1.1 & 0.977 & 791 & 0.026 & 0.058 & Accept $H_{0}$ \\
\hline 5 & & 15.5 & 45.0 & 5.2 & 0.695 & 126 & 0.051 & 0.15 & Accept $H_{0}$ \\
\hline 6 & $0.30-0.36$ & 11.1 & 40.2 & 2.2 & 0.917 & 323 & 0.036 & 0.091 & Accept $H_{0}$ \\
\hline 7 & & 12.3 & 40.0 & 2.3 & 0.898 & 302 & 0.053 & 0.094 & Accept $H_{0}$ \\
\hline 8 & & 14.8 & 38.4 & 2.1 & 0.916 & 224 & 0.036 & 0.11 & Accept $H_{0}$ \\
\hline 9 & $0.36-0.44$ & 12.3 & 44.1 & 1.7 & 0.955 & 500 & 0.029 & 0.073 & Accept $H_{0}$ \\
\hline 10 & & 13.2 & 38.0 & 2.0 & 0.929 & 318 & 0.034 & 0.091 & Accept $H_{0}$ \\
\hline 11 & & 14.4 & 44.4 & 2.2 & 0.925 & 374 & 0.026 & 0.084 & Accept $H_{0}$ \\
\hline 12 & & 15.8 & 34.2 & 1.8 & 0.931 & 269 & 0.033 & 0.099 & Accept $H_{0}$ \\
\hline 13 & & 17.2 & 33.0 & 1.7 & 0.926 & 372 & 0.096 & 0.085 & Reject $H_{0}$ \\
\hline
\end{tabular}

$A$ is the regression coefficient; $R^{2}$ is the correlation coefficient.

$D_{n}$ is test statistic; $D_{n, 0.01}$ is critical value of test statistic; $H_{0}$ : The sample data follow an exponential distribution. 

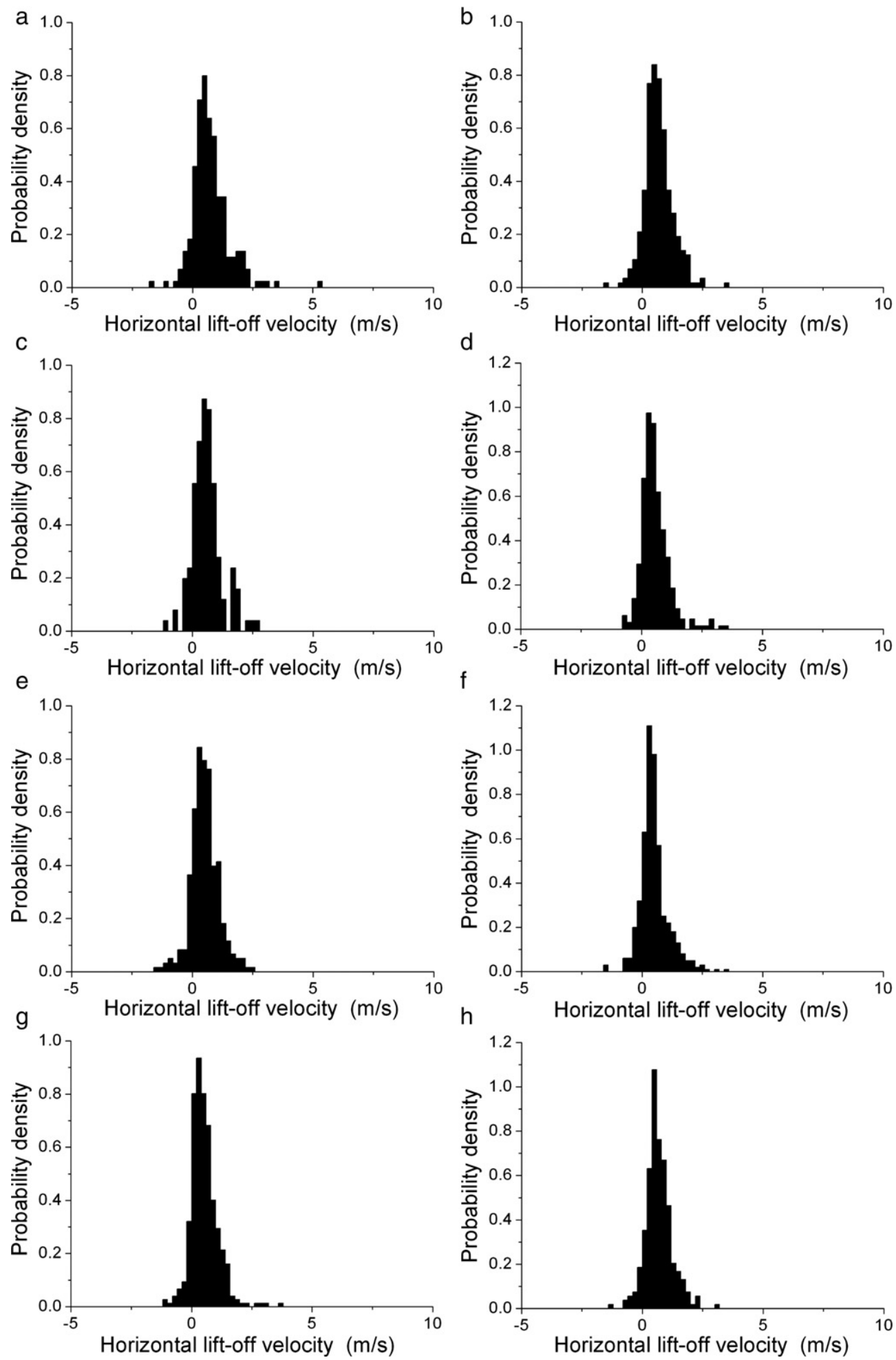

Fig. 2. The distribution of horizontal lift-off velocity of the saltating grains. ((a) $d_{p}=0.17-0.30 \mathrm{~mm}, u_{f 0}=10.9 \mathrm{~m} / \mathrm{s}$; (b) $d_{p}=0.17-0.30 \mathrm{~mm}$, $u_{f 0}=13.5 \mathrm{~m} / \mathrm{s}$; (c) $d_{p}=0.17-0.30 \mathrm{~mm}, u_{f 0}=15.5 \mathrm{~m} / \mathrm{s}$; (d) $d_{p}=0.30-0.36 \mathrm{~mm}, u_{f 0}=11.1 \mathrm{~m} / \mathrm{s}$; (e) $d_{p}=0.30-0.36 \mathrm{~mm}, u_{f 0}=12.3 \mathrm{~m} / \mathrm{s} ;(\mathrm{f}) d_{p}=0.36-$ $0.44 \mathrm{~mm}, u_{f 0}=12.3 \mathrm{~m} / \mathrm{s}$; (g) $d_{p}=0.36-0.44 \mathrm{~mm}, u_{f 0}=14.4 \mathrm{~m} / \mathrm{s}$; (h) $d_{p}=0.36-0.44 \mathrm{~mm}, u_{f 0}=15.8 \mathrm{~m} / \mathrm{s}$.) 

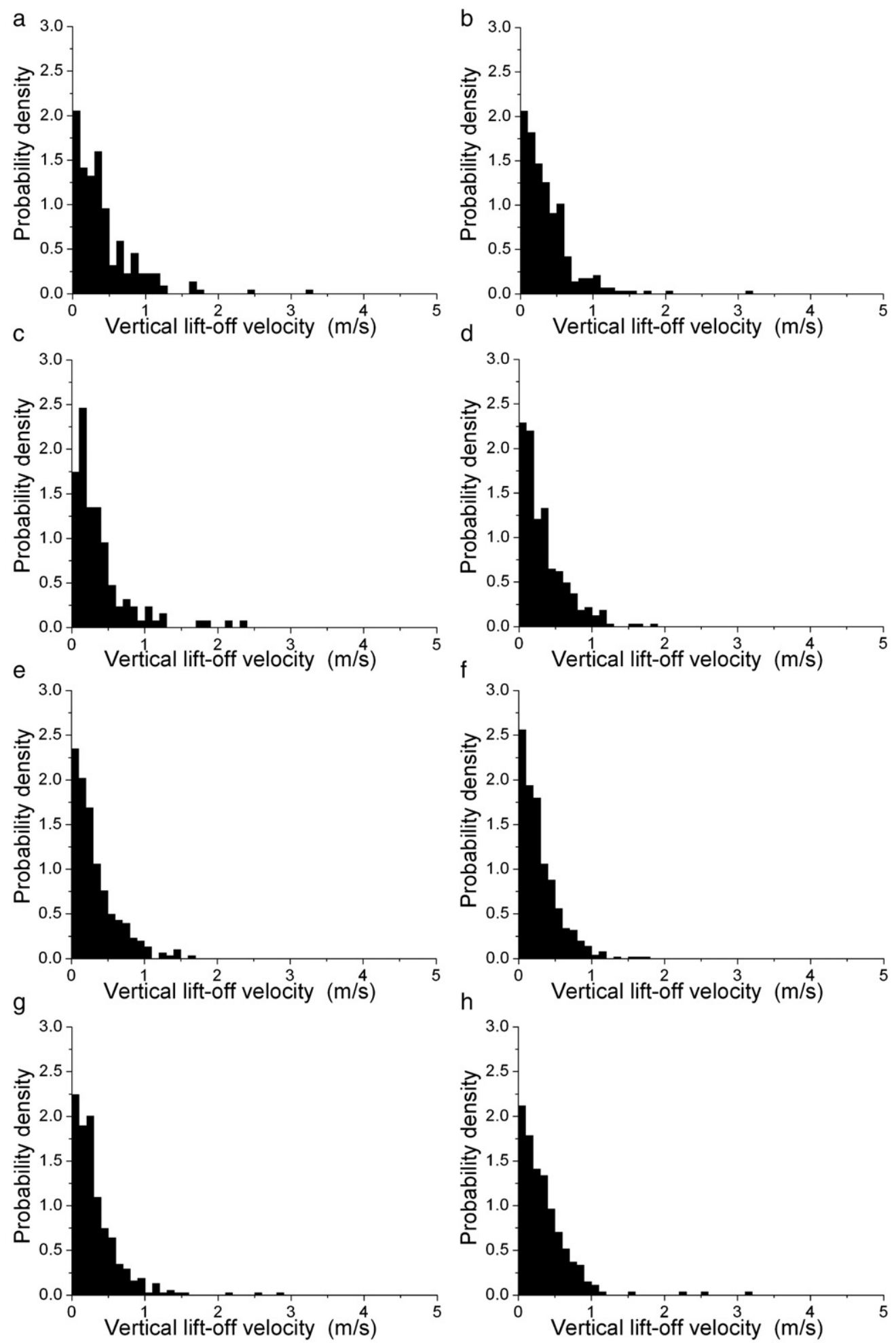

Fig. 3. The distribution of vertical lift-off velocity of the saltating grains. ((a) $d_{p}=0.17-0.30 \mathrm{~mm}, u_{f 0}=10.9 \mathrm{~m} / \mathrm{s} ;$ (b) $d_{p}=0.17-0.30 \mathrm{~mm}, u_{f 0}=13.5 \mathrm{~m} / \mathrm{s}$; (c) $d_{p}=0.17-0.30 \mathrm{~mm}, u_{f 0}=15.5 \mathrm{~m} / \mathrm{s}$; (d) $d_{p}=0.30-0.36 \mathrm{~mm}, u_{f 0}=11.1 \mathrm{~m} / \mathrm{s}$; (e) $d_{p}=0.30-0.36 \mathrm{~mm}, u_{f 0}=12.3 \mathrm{~m} / \mathrm{s}$; (f) $d_{p}=0.36-0.44 \mathrm{~mm}$, $u_{f 0}=12.3 \mathrm{~m} / \mathrm{s} ;(\mathrm{g}) d_{p}=0.36-0.44 \mathrm{~mm}, u_{f 0}=14.4 \mathrm{~m} / \mathrm{s}$; (h) $d_{p}=0.36-0.44 \mathrm{~mm}, u_{f 0}=15.8 \mathrm{~m} / \mathrm{s}$.) 
level $\alpha$ is set to 0.01 , the critical value of the test statistic is $D_{n, 0.01}=\frac{1.63}{\sqrt{n}}$ for $n>100$.

Tables $2^{\sqrt{n}}$ and 3 also show the results of the Kolmogorov-Smirnov test for the distributions of horizontal and vertical lift-off velocities of the saltating grains. It can be seen that, for the probability density function of horizontal lift-off velocity, the 12 tests are accepted within the total 13 tests. For the vertical liftoff velocity, there are also 12 tests accepted. The proportion of accepted tests, i.e., the accepted probability of specified distribution, is $92 \%$ for both horizontal and vertical lift-off velocities. Therefore, we can conclude that the probability distribution of horizontal lift-off velocity is a normal function and the vertical lift-off velocity follows an exponential distribution.

The present measurement results are consistent with the description in some analytical models (Sørensen, 1991; Raupach, 1991; Anderson and Hallet, 1986). Sørensen (1991) applied a normal distribution to describe the sand horizontal ejection velocity, and the exponential distribution of initial vertical particle velocity is used by Raupach (1991) and Anderson and Hallet (1986). The experiments of Nalpanis et al. (1993) show that the distribution of vertical lift-off velocity has a peak, but the statistical distribution pattern is not given. The movement of the lift-off grains on a bed surface is highly stochastic, however the present results provide a better description for the horizontal and vertical lift-off velocity distributions of the saltating grains.

\subsection{The distribution of resultant lift-off velocity and angle of the saltating grains}

Fig. 4 shows the probability density distribution of resultant lift-off velocity of the saltating grains. For any grain size at different free-stream wind velocities, the probability distribution can be described by a lognormal distribution.

The probability density function of resultant lift-off velocity of sand particles can be expressed as follows:

$P\left(u_{L}\right)=\frac{1}{\sqrt{2 \pi} A u_{L}} \exp \left(-\frac{\left(\ln u_{L}-\ln B\right)^{2}}{2 A^{2}}\right)$

where $P\left(u_{L}\right)$ is the probability density, $u_{L}$ is the resultant lift-off velocity of the saltating grains in meters per second. $A$ and $B$ are the regression coefficients.

Fig. 5 gives the probability density distribution of lift-off angle of the saltating grains. It can be seen that the probability of lift-off angle decreases with increasing angle. For any grain size at different free-stream wind velocities, the probability distribution can be expressed by an exponential function.

The probability density function of lift-off angle of the saltating grains is described by:

$P\left(\alpha_{L}\right)=\frac{1}{A} \exp \left(-\frac{\alpha_{L}}{A}\right)$

where $P\left(\alpha_{L}\right)$ is the probability density, $\alpha_{L}$ is the lift-off angle of the saltating grains in degrees. $A$ is the regression coefficient.

Tables 4 and 5 list the curve-fit parameters and Kolmogorov-Smirnov testing of the resultant lift-off velocity and angle distribution of the saltating grains, respectively. It can be seen that the correlation between the curve-fit function and the experimental data is reasonably good. The correlation coefficients of most fitting curves are more than 0.9 . For probability density function of resultant lift-off velocity, 11 tests are accepted within the total 13 tests. For the lift-off angle, 12 tests are accepted. The proportion of accepted tests is $85 \%$ for resultant lift-off velocity and $92 \%$ for lift-off angle. Therefore, the probability distribution of resultant lift-off velocity can be described by a lognormal function and the probability density function of lift-off angle can be expressed as an exponential function.

The wind tunnel experiment of White and Schulz (1977) shows the distributions of launch velocity and angle have a peak. Nalpanis et al. (1993) found that the probability distributions of resultant lift-off velocity and angle are similar to a $\log$-normal distribution. The experiment of Dong et al. (2002a) described the velocity distribution of entrained particles as a Weibull function, the probability of resultant lift-off velocity decreases with increasing resultant lift-off velocity, but the probability distribution of lift-off angles is complex and cannot be expressed by a simple function. In the numerical model of Namikas (2003), both gamma and exponential launch velocity distributions are studied. Therefore, the entrainment process is very complex, but the present experiments provide a reference for the distributions of resultant lift-off velocity and angle of the saltating grains.

In the present experiments, the backward lift-off of grains is detected. In Fig. 2, the negative value of horizontal lift-off velocity appears. In Fig. 5 there are some saltating particles whose lift-off angle is more than 90 degrees. Therefore, the particle collisions are intensive near the sand bed surface. 

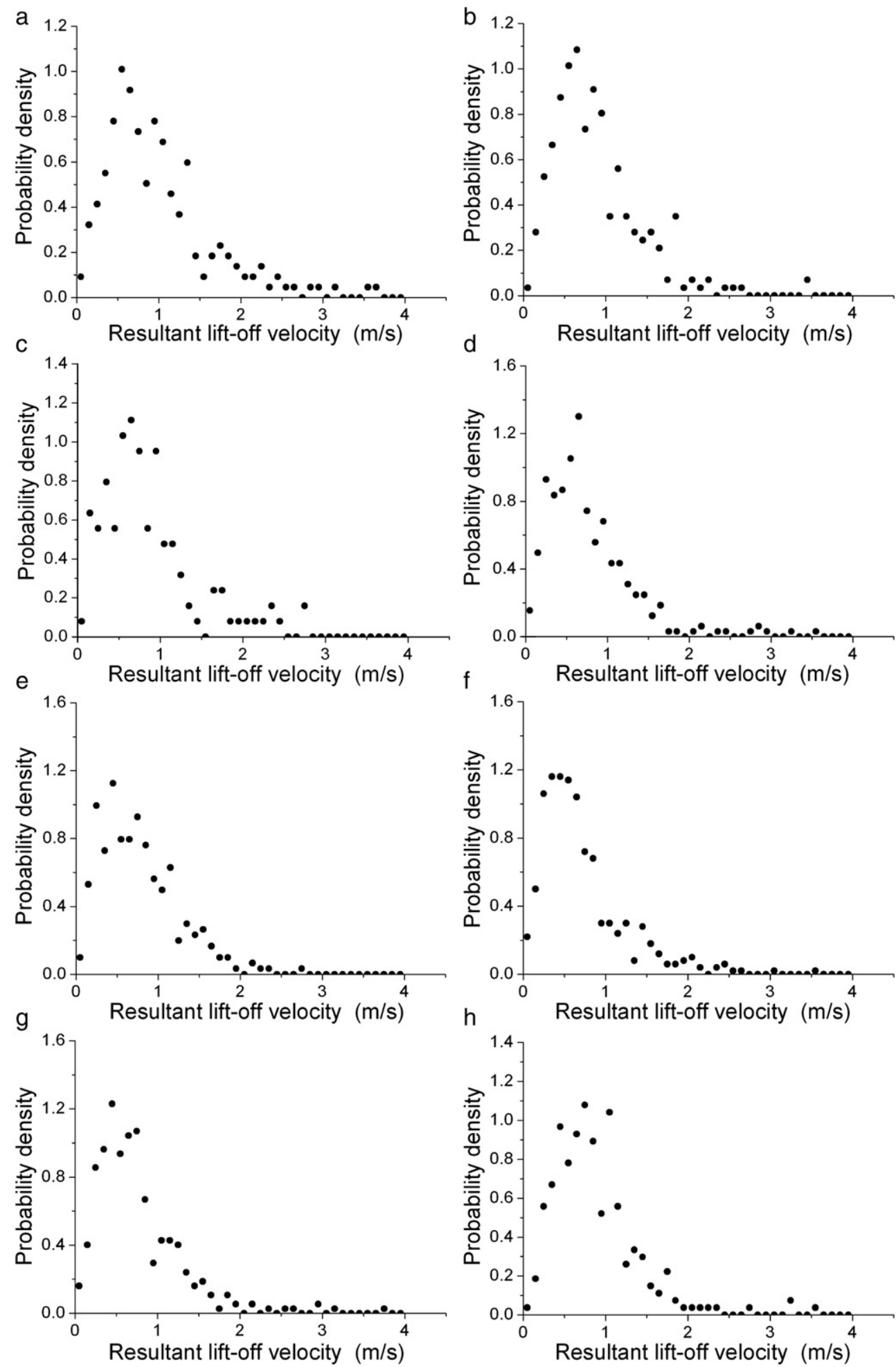

Fig. 4. The distribution of resultant lift-off velocity of the saltating grains. ((a) $d_{p}=0.17-0.30 \mathrm{~mm}, u_{f 0}=10.9 \mathrm{~m} / \mathrm{s}$; (b) $d_{p}=0.17-0.30 \mathrm{~mm}$, $u_{f 0}=13.5 \mathrm{~m} / \mathrm{s} ;$; (c) $d_{p}=0.17-0.30 \mathrm{~mm}, u_{f 0}=15.5 \mathrm{~m} / \mathrm{s}$; (d) $d_{p}=0.30-0.36 \mathrm{~mm}, u_{f 0}=11.1 \mathrm{~m} / \mathrm{s}$; (e) $d_{p}=0.30-0.36 \mathrm{~mm}, u_{f 0}=12.3 \mathrm{~m} / \mathrm{s} ;(\mathrm{f}) d_{p}=0.36-$ $0.44 \mathrm{~mm}, u_{f 0}=12.3 \mathrm{~m} / \mathrm{s}$; (g) $d_{p}=0.36-0.44 \mathrm{~mm}, u_{f 0}=14.4 \mathrm{~m} / \mathrm{s}$; (h) $d_{p}=0.36-0.44 \mathrm{~mm}, u_{f 0}=15.8 \mathrm{~m} / \mathrm{s}$.) 

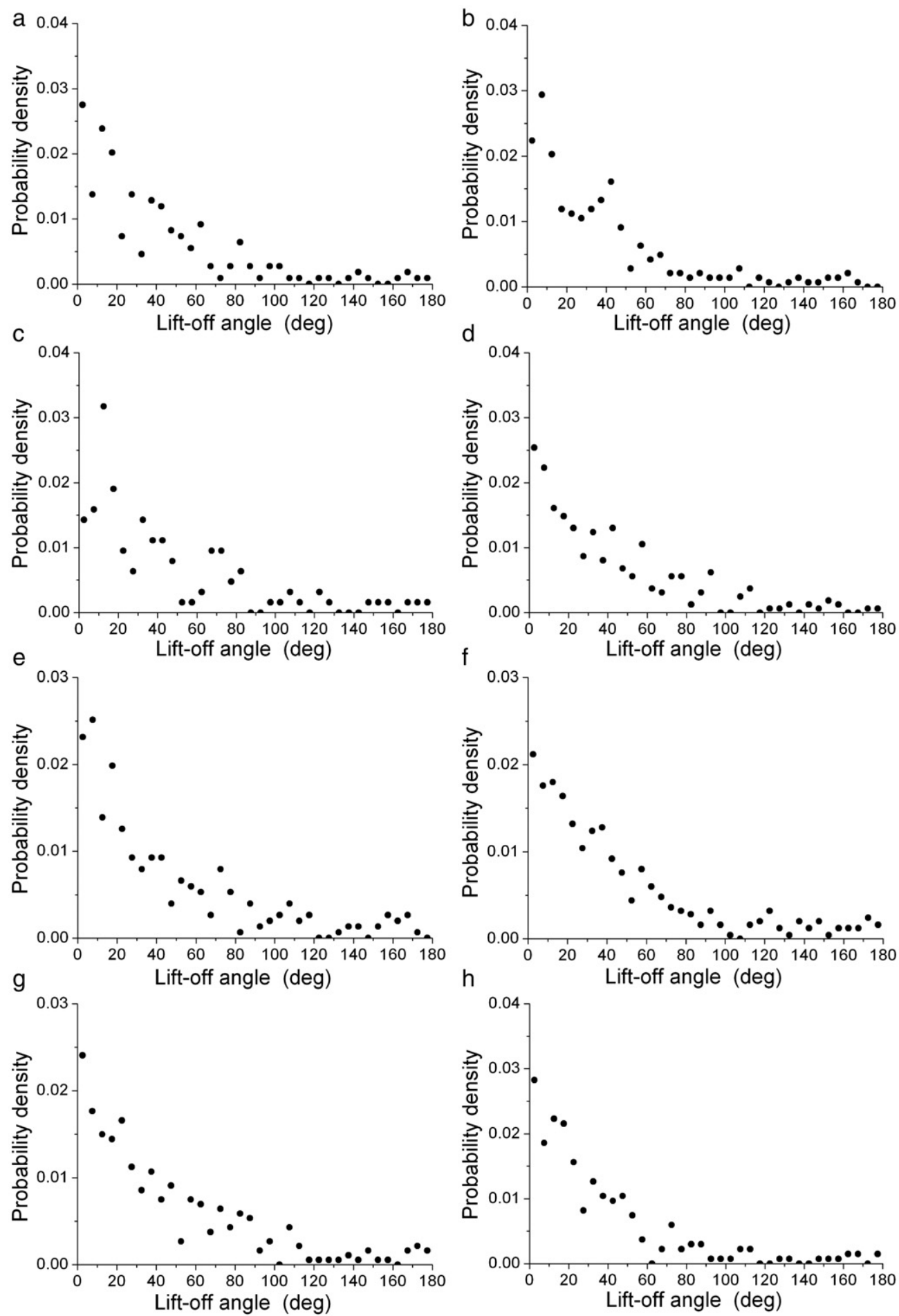

Fig. 5. The distribution of lift-off angle of the saltating grains. ((a) $d_{p}=0.17-0.30 \mathrm{~mm}, u_{f 0}=10.9 \mathrm{~m} / \mathrm{s} ;$ (b) $d_{p}=0.17-0.30 \mathrm{~mm}, u_{f 0}=13.5 \mathrm{~m} / \mathrm{s}$; (c) $d_{p}=0.17-0.30 \mathrm{~mm}, u_{f 0}=15.5 \mathrm{~m} / \mathrm{s}$; (d) $d_{p}=0.30-0.36 \mathrm{~mm}, u_{f 0}=11.1 \mathrm{~m} / \mathrm{s}$; (e) $d_{p}=0.30-0.36 \mathrm{~mm}, u_{f 0}=12.3 \mathrm{~m} / \mathrm{s}$; (f) $d_{p}=0.36-0.44 \mathrm{~mm}$, $u_{f 0}=12.3 \mathrm{~m} / \mathrm{s} ;(\mathrm{g}) d_{p}=0.36-0.44 \mathrm{~mm}, u_{f 0}=14.4 \mathrm{~m} / \mathrm{s} ;(\mathrm{h}) d_{p}=0.36-0.44 \mathrm{~mm}, u_{f 0}=15.8 \mathrm{~m} / \mathrm{s}$.) 


\section{A numerical model for the vertical distribution of aeolian mass flux}

Most numerical models of aeolian sand transport are generally subdivided into four distinct subprocesses: aerodynamic entrainment, grain trajectories, grain-bed impacts, and wind field modification (Anderson and Haff, 1988, 1991; Ungar and Haff, 1987; McEwan and Willetts, 1991, 1993; Spies et al., 2000; Spies and McEwan, 2000). By linking these subprocesses, a negative feedback mechanism is established to form a saltating equilibrium state.

The model proposed in this paper is to investigate the fully developed flow of blowing sands. The number of grains due to aerodynamic entrainment can become rare at steady state (Anderson and Haff, 1988, 1991). Hence, the aerodynamic entrainment is neglected. The present numerical model includes three major components: the wind field, the grain trajectories and the grain-bed collisions.

\subsection{Wind field}

Assuming the wind field is a two-dimensional steady and horizontal shear flow, and the mixing length theory is used to calculate the effective viscosity, then the wind velocity can be expressed as follows (Anderson and Haff, 1991):

$\frac{\partial u_{a}}{\partial y}=\frac{1}{k y}\left(\frac{\tau_{0}-\tau_{p}}{\rho_{a}}\right)^{1 / 2}$

where

$\tau_{p}(y)=\int_{y}^{y_{\max }} F_{d x}(z) d z$

$\tau_{0}=\tau_{a}(y)+\tau_{p}(y)$

where $u_{a}$ is air velocity, $\rho_{a}$ is air density, $\tau_{0}$ is the total shear stress, $\tau_{a}$ is the airborne shear stress, $\tau_{p}$ is the grainborne shear stress, $k$ is von Karman's constant $(k=0.4), y$ is the height, $y_{\max }$ is the maximum saltation height of a saltating grain, and $F_{d x}$ is the volumetric fluid-particle drag force in the $x$ direction.

Owen (1964) hypothesized that the airborne shear stress at the ground in the equilibrium saltation can be given as:

$\tau_{a}(0)=\rho_{a} u_{* c}^{2}$

where $\tau_{a}(0)$ is the airborne shear stress at the ground and $u *_{c}$ is the threshold shear velocity.
The total shear stress $\tau_{0}$ is:

$\tau_{0}=\rho_{a} u_{*}^{2}$

where $u *$ is the shear velocity in the grain-free wind above the saltation cloud.

Then the grain-borne shear stress at the ground $\tau_{p}(0)$ is:

$\tau_{p}(0)=\rho_{a}\left(u_{*}^{2}-u_{* c}^{2}\right)$

\subsection{Grain trajectories}

The gravitational and fluid drag forces are only considered for the calculation of grain trajectories. The equation of particle motion is:

$\frac{d \mathrm{u}_{p}}{d t}=\frac{f}{\tau_{v}}\left(\mathrm{u}_{a}-\mathrm{u}_{p}\right)+\mathrm{g}$

where $\mathbf{u}_{a}$ and $\mathbf{u}_{p}$ are the air and particle velocity vector, respectively, $\mathbf{g}$ is acceleration due to gravity, $f$ is the drag coefficient, and $\tau_{v}$ is the particle velocity response time, as follows:

$f=\left\{\begin{array}{ll}1+0.15 \mathrm{Re}_{p}^{0.687}, & \operatorname{Re}_{p}<1000 \\ 0.44 \frac{\operatorname{Re}_{p}}{24}, & \operatorname{Re}_{p} \geq 1000\end{array}, \quad \tau v=\frac{\rho_{p} d_{p}^{2}}{18 \mu}, \quad \operatorname{Re}_{p}=\frac{\rho_{a} d_{p}\left|u_{a}-u_{p}\right|}{\mu}\right.$

where $R e_{p}$ is the particle Reynolds number, $\rho_{p}$ is sand density, $d_{p}$ is the sand diameter, and $\mu$ is the fluid dynamic viscosity.

\subsection{Grain-bed collisions}

The grain-bed collision process determines the liftoff velocity and angle of blown sands on the bed surface, which further affects particle trajectories, wind field and sand mass flux. The treatments of the process are generally described by the splash function (Anderson and Haff, 1988, 1991; Ungar and Haff, 1987) or a set of the experimental data (McEwan and Willetts, 1991, 1993) to create the lift-off velocity and angle distribution of saltation grains.

In the present model, the probability distributions of sand lift-off velocity are given from our wind tunnel experiments.

According to Eqs. (1) and (2), the probability density functions of horizontal lift-off velocity of the entrained particles and of vertical lift-off velocity can be expressed as follows, respectively:

$P_{u}\left(u_{0}\right)=\frac{1}{\sqrt{2 \pi} \sigma} \exp \left[-\frac{\left(u_{0}-<u_{0}>\right)^{2}}{2 \sigma^{2}}\right]$ 
$P_{v}\left(v_{0}\right)=\frac{1}{<v_{0}>} \exp \left(\frac{-v_{0}}{<v_{0}>}\right)$

where $P_{u}$ and $P_{v}$ are the probability density, and $u_{0}$ and $v_{0}$ are the horizontal and vertical lift-off velocities of the saltating grains, respectively. $\left\langle u_{0}\right\rangle$ and $\left\langle v_{0}\right\rangle$ are the average horizontal and vertical lift-off velocities, respectively, and $\sigma$ is the standard deviation of the horizontal lift-off velocity.

According to Eqs. (4) and (5), the probability density functions of resultant lift-off velocity and of lift-off angle are given as follows, respectively:

$P_{u l}\left(u_{L}\right)=\frac{1}{\sqrt{2 \pi} D u_{L}} \exp \left(-\frac{\left(\ln u_{L}-\ln C\right)^{2}}{2 D^{2}}\right)$

$P_{\alpha}\left(\alpha_{L}\right)=\frac{1}{B} \exp \left(-\frac{1}{B} \alpha_{L}\right)$

where $P_{u l}$ and $P_{\alpha}$ are the probability density, $u_{L}$ and $\alpha_{L}$ are the resultant lift-off velocity and angle, respectively. $B$ is the mean lift-off angle, $C$ is the median resultant lift-off velocity and $D$ means the standard deviation of resultant lift-off velocity's logarithm.

Using the probability density functions of horizontal and vertical lift-off velocities, the volumetric fluidparticle drag force $F_{d x}$ is:

$$
\begin{aligned}
F_{d x}(y)= & m n_{\uparrow}(0)<v_{\uparrow}(0)> \\
& \int_{-\infty}^{+\infty} P_{u}\left(u_{0}\right) \int_{0}^{\infty} P_{v}\left(v_{0}\right) \\
& \times\left[\frac{f_{d x \uparrow}\left(y, v_{0}, u_{0}\right)}{v_{\uparrow}\left(y, v_{0}, u_{0}\right)}-\frac{f_{d x \downarrow}\left(y, v_{0}, u_{0}\right)}{v_{\downarrow}\left(y, v_{0}, u_{0}\right)}\right] d v_{0} d u_{0}
\end{aligned}
$$

where the subscript $\uparrow$ and $\downarrow$ denote the ascending and descending grains, respectively. $m$ is the particle mass, $n$ is the number density of grains, and $v$ is the vertical velocity of grains. $f_{d x}=\frac{f}{\tau_{v}}\left(u_{a}-u_{p}\right)$, where $u_{a}$ and $u_{p}$ are the air and particle velocities at the $\mathrm{x}$ direction, respectively.

The sand mass flux can be expressed as:

$$
\begin{aligned}
q(y)= & m n_{\uparrow}(0)<v_{\uparrow}(0)>\int_{-\infty}^{+\infty} P_{u}\left(u_{0}\right) \\
& \int_{0}^{\infty} P_{v}\left(v_{0}\right)\left[\frac{u_{\uparrow}\left(y, v_{0}, u_{0}\right)}{v_{\uparrow}\left(y, v_{0}, u_{0}\right)}-\frac{u_{\downarrow}\left(y, v_{0}, u_{0}\right)}{v_{\downarrow}\left(y, v_{0}, u_{0}\right)}\right] d v_{0} d u_{0}
\end{aligned}
$$

where $u$ is the horizontal velocity of the grains and $q$ is the sand mass flux.
Using the probability density functions of resultant lift-off velocity and angle, we can get

$$
\begin{aligned}
F_{d x}(y)= & m n_{\uparrow}(0)<v_{\uparrow}(0)> \\
& \int_{0}^{+\infty} P_{u l}\left(u_{L}\right) \int_{0}^{\pi} P_{\alpha}\left(\alpha_{L}\right) \\
& \times\left[\frac{f_{d x \uparrow}\left(y, u_{L}, \alpha_{L}\right)}{v_{\uparrow}\left(y, u_{L}, \alpha_{L}\right)}-\frac{f_{d x \downarrow}\left(y, u_{L}, \alpha_{L}\right)}{v_{\downarrow}\left(y, u_{L}, \alpha_{L}\right)}\right] d \alpha_{L} d u_{L} \\
q(y)= & m n_{\uparrow}(0)<v_{\uparrow}(0)>\int_{0}^{+\infty} P_{u l}\left(u_{L}\right) \int_{0}^{\pi} P_{\alpha}\left(\alpha_{L}\right) \\
& \times\left[\frac{u_{\uparrow}\left(y, u_{L}, \alpha_{L}\right)}{v_{\uparrow}\left(y, u_{L}, \alpha_{L}\right)}-\frac{u_{\downarrow}\left(y, u_{L}, \alpha_{L}\right)}{v_{\downarrow}\left(y, u_{L}, \alpha_{L}\right)}\right] d \alpha_{L} d u_{L}
\end{aligned}
$$

\section{Computational conditions and methods}

Namikas (2003) measured the vertical distribution of aeolian mass flux. The present simulation of aeolian mass flux is compared with the field data of Namikas (2003).

Here, two cases are evaluated, i.e., one uses the Eqs. (13) and (14), the other uses Eqs. (15) and (16). The setting parameters are listed in the following:

(1) Case 1 (using the Eqs. (13) and (14)):

The diameter of sand is $0.25 \mathrm{~mm}$, and its density is $2650 \mathrm{~kg} / \mathrm{m}^{3}$. The average horizontal lift-off velocity is $0.7 \mathrm{~m} / \mathrm{s}$, the standard deviation of the horizontal lift-off velocity $\sigma$ is $0.4 \mathrm{~m} / \mathrm{s}$. The average vertical lift-off velocity is $0.5 \mathrm{~m} / \mathrm{s}$.

(2) Case 2 (using the Eqs. (15) and (16)):

The diameter of sand is $0.25 \mathrm{~mm}$, and its density is $2650 \mathrm{~kg} / \mathrm{m}^{3}$. The median resultant lift-off velocity is $0.86 \mathrm{~m} / \mathrm{s}$, the D in Eq. (15) is 0.56 . The mean lift-off angle is $35^{\circ}$.

All simulations of Case 1 include 5535 trajectories. Each simulation of Case 2 involves 5040 trajectories. The contributions of each trajectory are weighted according to the combined probability acquired from the given probability density function of lift-off velocity. The simulated sand mass flux is determined by summing the contributions of each trajectory.

The present numerical model is used to simulate the relative proportion of sand mass flux as a function of height. Therefore, the simulated mass flux is scaled by the total measured mass flux for comparison with the field data of Namikas (2003).

In fact, the simulation is also dependent on the assumption that the grains leaving the ground follow the same distribution of trajectories on average. 


\section{Computational results and discussions}

Using the numerical model of this paper, the vertical distributions of sand mass flux are computed.

Many studies have revealed that there is an exponential decay of mass flux with height (Dong et al., 2002b; Greeley et al., 1996; Rasmussen and Sørensen, 1999). The experiment of Dong et al. (2002b) shows the sand mass flux decreases exponentially with height. The field measurement of Greeley et al. (1996) shows that there is a clear log-linear decrease in sand flux with height. Rasmussen and Sørensen (1999) used the isokinetic trap to measure the sand mass flux in a field, and there is an exponential behavior between mass flux and height.

The simulated mass flux of this paper and the field data of Namikas (2003) are plotted in a log-linear coordinate system, shown in Fig. 6. In Fig. $6 q$ is the sand mass flux and $u *$ is the shear velocity. We can see that the vertical distribution of mass flux is an exponential distribution, except at the lower height. In
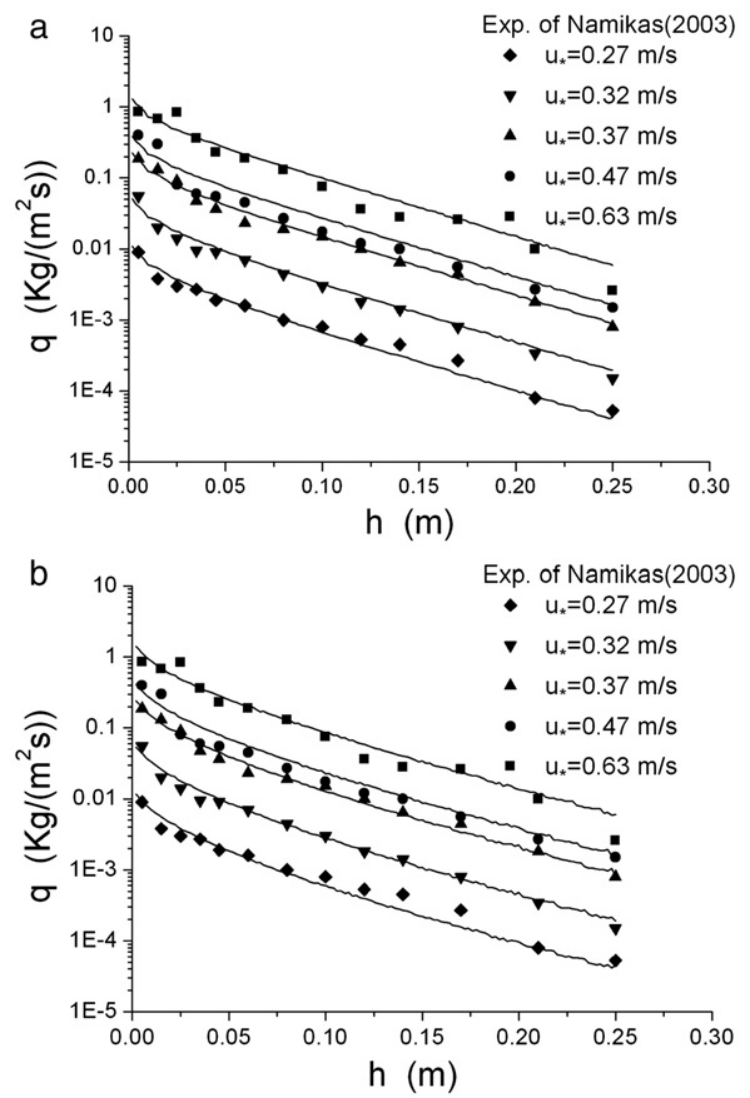

Fig. 6. Comparison of the simulated (lines) sand mass flux with the measured (symbols) data of Namikas (2003). ((a) Case 1; (b) Case 2.)
Table 6

The comparison of the relative decay rate

\begin{tabular}{llllll}
\hline$u_{*(\mathrm{~m} / \mathrm{s})}$ & $b_{\exp }$ & $b_{c 1}$ & $\begin{array}{l}\left(b_{c 1}-b_{\exp }\right) / b_{\exp } \\
(\%)\end{array}$ & $b_{c 2}$ & $\begin{array}{l}\left(b_{c 2}-b_{\exp }\right) / b_{\exp } \\
(\%)\end{array}$ \\
\hline 0.27 & 18.4 & 19.75 & 7.3 & 19.85 & 7.9 \\
0.32 & 21.5 & 19.47 & -9.4 & 19.74 & -8.2 \\
0.37 & 20.4 & 19.4 & -9.4 & 19.67 & -3.6 \\
0.47 & 19.6 & 19.37 & -1.2 & 19.5 & -0.5 \\
0.63 & 22.2 & 19.12 & -13.9 & 19.38 & -12.7 \\
\hline
\end{tabular}

addition, the simulated mass flux is a good fit to the field results of Namikas (2003) across the entire height.

From Fig. 6, all simulated vertical profiles of mass flux in the near-bed region deviate from the exponential distribution. This deviation from an exponential profile is also reported by some published experimental data (e.g., Ni et al., 2002). In fact, the mass flux distribution is related to the different modes of sand transport. The saltation grains are generally responsible for the exponential distribution of sand mass flux, and the creep grains probably introduce the additional near-bed mass flux and lead to the deviation from the exponential distribution in the near-bed region.

The exponential distribution of sand mass flux can be described as the following function:

$q=a \exp (-b h)$

where $q$ is the sand mass flux at height $h$, in kilograms per square meter per second, $h$ is the height in meters. $a$ and $b$ are the coefficients.

In Eq. (21), the coefficient $b$ denotes the relative decay rate of the sand mass flux with height. By using the least squares curve-fit method for the simulated mass flux at $h>0.05 \mathrm{~m}$, the relative decay rate $b$ of Cases 1 and 2 is acquired and is shown in Table 6.

It can be seen from Table 6 that the simulated relative decay rate falls within the experimental data of Namikas (2003) at different shear velocities $u *$. The agreement is good. Therefore, the lift-off velocity distributions from this present wind tunnel experiment are able to predict the sand mass flux.

In Table $6, u *$ is wind shear velocity. $b_{\text {exp }}, b_{c 1}$ and $b_{c 2}$ are the relative decay rate of sand mass flux in experiments of Namikas (2003), Case 1 and Case 2, respectively.

From Table 6, it also can be seen that in Case 1 and 2, the simulated relative decay rate of the sand mass flux decreases with the increase in shear velocity $u *$. This is consistent with the wind tunnel experiments of Dong et al. (2002b). In the analysis of Dong et al. (2002b), the 
relative decay rate decreases with the increase in freestream wind velocity, but the shear velocity is not reported. That is to say, when the wind strength increases, the relative decay rate will decrease. This also implies that more sand grains at the lower height are transported to the higher height as the wind velocity increases.

\section{Conclusions}

The lift-off velocity of the saltating grains is measured by wind tunnel experiment. The probability distributions of lift-off velocity in aeolian sand transport are found as follows:

(1) The probability density function of horizontal liftoff velocity of the saltating grains is a normal function, and that of vertical lift-off velocity is an exponential function.

(2) The probability density function of resultant liftoff velocity of the saltating grains can be expressed as a log-normal function, and that of lift-off angle complies with an exponential function.

According to the lift-off velocity distributions from the wind tunnel experiment in this paper, a numerical model is established to reconstruct the vertical distribution of saltation mass flux. The simulated saltation mass flux distribution is consistent with the field data of Namikas (2003). Therefore, the present investigations are helpful in understanding the probability characteristics of the lift-off state of grains in windblown sand movement.

Accurately describing the lift-off velocity distribution is important in studying the macroscopic parameters such as sand mass flux, and the lift-off velocity distribution is a bridge to linking the microscopic and macroscopic aeolian research. The present work supplies an interesting reference for the study of liftoff velocity.

The entrainment process in saltation is complex. The particle collisions are intensive near the sand bed surface. In the present experiment, the backward liftoff of grains is detected. The mid-air collisions may bring a favorable condition for the generation of the backward lift-off grains.

\section{Acknowledgements}

This work is supported by National Natural Science Foundation of China (Grant No. 10532030) and Major
State Basic Research Development Program of China (Grant No. G2000048702).

\section{References}

Anderson, R.S., Haff, P.K., 1991. Wind modification and bed response during saltation of sand in air. Acta Mechanica (suppl.1), 21-51.

Anderson, R.S., Hallet, B., 1986. Sediment transport by wind: toward a general model. Geological Society of America Bulletin 97, 523-535.

Anderson, R.S., Haff, P.K., 1988. Simulation of eolian saltation. Science 241, 820-823.

Bagnold, R.A., 1941. The Physics of Blown Sand and Desert Dunes. Methuen, London.

Dong, Z.B., Liu, X.P., Li, F., Wang, H.T., Zhao, A.G., 2002a. Impactentrainment relationship in a saltating cloud. Earth Surface Processes and Landforms 27, 641-658.

Dong, Z.B., Liu, X.P., Wang, H.T., Zhao, A.G., Wang, X.M., 2002b. The flux profile of a blowing sand cloud: a wind tunnel investigation. Geomorphology 49, 219-230.

Greeley, R., Iversen, J.D., 1985. Wind as a Geological Process on Earth, Mars, Venus and Titan. Cambridge Univ. Press, Cambridge.

Greeley, R., Blumberg, D.G., Williams, S.H., 1996. Field measurements of the flux and speed of wind-blown sand. Sedimentology $43,41-52$.

Haff, P.K., Anderson, R.S., 1993. Grain scale simulations of loose sedimentary beds: the example of grain-bed impacts in aeolian saltation. Sedimentology 40, 175-198.

McEwan, I.K., Willetts, B.B., 1991. Numerical model of the saltation cloud. Acta Mechanica (suppl.1), 53-66.

McEwan, I.K., Willetts, B.B., 1993. Adaptation of the near-surface wind to the development of sand transport. Journal of Fluid Mechanics 252, 99-105.

Mitha, S., Tran, M.Q., Werner, B.T., Haff, P.K., 1986. The grain-bed impact process in aeolian saltation. Acta Mechanica 63, 267-278.

Nalpanis, P., Hunt, J.C.R., Barrett, C.F., 1993. Saltating particles over flat beds. Journal of Fluid Mechanics 251, 661-685.

Namikas, S.L., 2003. Field measurement and numerical modelling of aeolian mass flux distributions on a sandy beach. Sedimentology 50, 303-326.

Ni, J.R., Li, Z.S., Mendoza, C., 2002. Vertical profiles of aeolian sand mass flux. Geomorphology 49, 205-218.

Owen, P.R., 1964. Saltation of uniform grains in air. Journal of Fluid Mechanics 20, 225-242.

Rasmussen, K.R., Sørensen, M., 1999. Aeolian mass transport near the saltation threshold. Earth Surface Processes and Landforms 24, 413-422.

Raupach, M.R., 1991. Saltation layers. Vegetation canopies and roughness lengths. Acta Mechanica (Suppl.1), 83-96.

Rice, M.A., Willetts, B.B., McEwan, I.K., 1995. An experimental study of multiple grain-size ejecta produced by collisions of saltating grains with a flat bed. Sedimentology 42, 695-706.

Rice, M.A., Willetts, B.B., McEwan, I.K., 1996. Observation of collisions of saltating grains with a granular bed from high-speed cine-film. Sedimentology 43, 21-31.

Spies, P.-J., McEwan, I.K., Butterfield, G.R., 2000. One-dimentional transitional behaviour in saltation. Earth Surface Processes and Landforms 25, 505-518.

Spies, P.-J., McEwan, I.K., 2000. Equilibration of saltation. Earth Surface Processes and Landforms 25, 437-453. 
Sørensen, M., 1991. An analytic model of wind-blown sand transport. Acta Mechanica (Suppl.1), 67-81.

Ta, W.Q., Dong, Z.B., 2007. Simulation on sand grain/bed collision mechanism: cascade collision and ejection(1). Geomorphology 89, 348-357.

Ungar, J.E., Haff, P.K., 1987. Steady state saltation in air. Sedimentology 34, 289-299.

Werner, B.T., Haff, P.K., 1988. The impact process in aeolian saltation: two-dimensional simulations. Sedimentology 35, 189-196.

White, B.R., Schulz, J.C., 1977. Magnus effect on saltation. Journal of Fluid Mechanics 81, 497-512.
Willetts, B.B., Rice, M.A., 1986. Collisions in aeolian saltation. Acta Mechanica 63, 255-265.

Zhang, W., Kang, J.H., Lee, S.J., 2007a. Tracking of saltating sand trajectories over a flat surface embedded in an atmospheric boundary layer. Geomorphology 86, 320-331.

Zhang, W., Wang, Y., Lee, S.J., 2007b. Two-phase measurements of wind and saltating sand in an atmospheric boundary layer. Geomorphology 88, 109-119.

Zou, X.Y., Wang, Z.L., Hao, Q.Z., Zhang, C.L., Liu, Y.Z., Dong, G.R., 2001. The distribution of velocity and energy of saltating sand grains in a wind tunnel. Geomorphology $36,155-165$. 